\title{
Efectividad de un Curso de Capacitación en un Ambiente Virtual de Aprendizaje
}

\section{Effectiveness of a Training Course in a Virtual Learning Environment}

\author{
Marco Antonio CHAVEZ ARCEGA y Liz ROMERO \\ Universidad Autónoma de Nayarit (México) y Nova Southeastern University (EEUU)
}

Recibido: Noviembre 2011

Aceptado: Febrero 2012

\section{Resumen}

El estudio determinó la efectividad de un curso de capacitación impartido en un Ambiente Virtual de Aprendizaje (AVA). Utilizando el modelo de Kirkpatrick se midió la efectividad, considerando como variables el aprendizaje, la satisfacción, la aplicabilidad de los conocimientos, y los resultados alcanzados por 25 participantes. Se hicieron mediciones con diferentes instrumentos de recolección de información. El análisis de los datos indicó que los conocimientos de los estudiantes aumentaron, se sintieron satisfechos, aplicaron los conocimientos adquiridos, y se alcanzaron los resultados esperados. Se concluyó que el curso en el AVA fue efectivo.

Palabras clave: Efectividad, Capacitación Docente, Aprendizaje Virtual, Modelo Kirkpatrick.

\begin{abstract}
The study determined the effectiveness of a training course taught in a Virtual Learning Environment (VLE). Using Kirkpatrick's model effectiveness was measured, considering the learning, the satisfaction, the applicability of knowledge, and the results achieved by 25 participants. Measurements were made with different data collection instruments. The analysis of the data indicated that: the knowledge of students increased, they were satisfied, applied knowledge, and achieved the expected results. It was concluded that the course was effective in the VLE.
\end{abstract}

Keywords: Effectiveness, Teacher Training, Virtual Learning, Model Kirkpatrick.

El presente estudio involucró un proceso de capacitación docente, por lo que es necesario definir el concepto de capacitación, para diferenciarlo del entrenamiento y el adiestramiento.

La capacitación es la adquisición de conocimientos técnicos, teóricos y prácticos que van a contribuir al desarrollo de individuos en el desempeño de una actividad. En cambio, el entrenamiento es la preparación que se sigue para desempeñar una función, 
mientras que el adiestramiento es el proceso mediante el cual se estimula al trabajador a incrementar sus conocimientos, destrezas y habilidades (Soria, 2006).

\section{La Capacitación Docente}

La capacitación del docente debe concebirse como un proceso permanente, que conlleva en su desarrollo amplios debates académicos. El mejoramiento del trabajo docente, no depende únicamente de los programas de capacitación y actualización; aunque sí pueden contribuir a que los profesores reflexionen acerca de su inserción en el tiempo que les ha tocado vivir, para que participen de una manera más protagónica en la construcción de un porvenir mejor, tarea que redundará en la construcción de una pedagogía basada en el conocimiento profundo, en la que deberán emplear diversos y nuevos métodos para llevar a cabo el aprendizaje (Almanzor, 2007).

\section{La Capacitación Docente a Distancia}

Las formas de capacitación docente se han diversificado. En años recientes, cada día son más los programas de capacitación docente que emigran de una modalidad presencial a una modalidad de capacitación en línea (Mendoza, 2003). Los avances tecnológicos han permitido hacer una realidad la modalidad de educación a distancia. La capacitación docente a distancia presenta diversas ventajas como los son la flexibilidad de horarios, el ahorro de tiempos de desplazamiento, la posibilidad de estudiar varias veces el material hasta comprenderlo, la ayuda de tutores en línea, la disminución de costos, la autonomía del aprendizaje, el trabajo colaborativo, entre otras (Mendoza, 2003).

\section{La Capacitación a Distancia y las Buenas Prácticas}

La capacitación a distancia debe ser precedida por buenas prácticas, tal y como lo señala el New York State Education Department (2007) que sugiere que toda institución educativa que ofrece educación a distancia debe tener un compromiso organizacional, que garantice la calidad y eficacia en todos los aspectos del aprendizaje. Así mismo, esta organización considera que las instituciones que ofrecen educación a distancia, deben proponer diseños instruccionales acordes a dicha modalidad, evaluar sus programas, ofrecer capacitación permanente a su personal académico, configurar las metas de aprendizaje presenciales igual que las metas de aprendizaje a distancia, definir las buenas prácticas en las que llevarán a cabo la instrucción, entre otros aspectos.

En el mismo sentido, Sorensen \& Baylen (2009) señalan que las buenas prácticas de la instrucción a distancia, estimulan la comunicación entre estudiantes y profesores, facilitan e incentivan el trabajo colaborativo ente los estudiantes, propician actividades para el aprendizaje, mejoran la retroalimentación académica, enfatizan en el tiempo de las asignaciones, propician altas expectativas de comunicación, y contribuyen a la demostración del talento por parte del estudiante.

\section{El Diseño de Cursos de Capacitación y los Ambientes Virtuales de Aprendizaje}

El diseño instruccional hace posible generar cursos de capacitación en línea que permiten aprender a aprender, a generar comunidades de aprendizaje, a fomentar la formación continua, a aprender en forma autónoma, a promover el interés por el aprendizaje, y al aprendizaje solidario (Señas, 2005). Según Smaldino, Lowther \& Russell 
(2008) un modelo de diseño instruccional que favorece lo destacado por Señas, es el modelo ASSURE, el cual de acuerdo a estos autores, representa una buena alternativa para el diseño de cursos a distancia.

Otro modelo de diseño instruccional para cursos a distancia es el propuesto por Dorrego \& García (1991) el cual se fundamenta en la teoría del procesamiento de la información y la teoría instruccional de Gagné.

Con respecto a los modelos de diseño instruccional Simonson, Smaldino, Albright \& Zvacek (2009) señalan que uno de los más utilizados, en los últimos años, es el de Dick, Carey \& Carey. Este modelo funciona en forma sistemática considerando las siguientes etapas: a) identificación de metas, b) análisis del aprendiz, c) identificación de objetivos, d) desarrollo y evaluación de instrumentos, e) desarrollo de estrategias instruccionales, f) desarrollo y selección de material instruccional, y g) la evaluación (Dick, Carey \& Carey, 2009).

Estos y otros modelos de diseño instruccional, han permitido organizar la instrucción utilizando las diferentes herramientas que conforman los AVAs. Lo cual a su vez ha contribuido a que poco a poco, los AVAs se hayan convertido en una alternativa para ofrecer capacitación (Chen \& Seong, 2005).

\section{Cursos de Capacitación en Ambientes Virtuales de Aprendizaje}

Existen muchas experiencias de desarrollo de cursos de capacitación utilizando los AVAs. Una de estas experiencias es la presentada por Grisolía \& Corral (2005), quienes llevaron a cabo el desarrollo de un AVA, para un curso sobre el aprendizaje del idioma inglés, ofrecido a docentes; los resultados obtenidos permitieron establecer que un curso en un ambiente de aprendizaje virtual puede ser la respuesta para la capacitación docente, en diversas áreas del conocimiento. Dichos autores recomendaron implantar programas de capacitación docente e investigar la eficiencia y efectividad de dichos programas.

Otra evidencia de capacitación a través de AVAs es la de Hamada (2007) quien describe el desarrollo de un ambiente virtual de aprendizaje (plataforma, materiales instruccionales, diseño del curso, entre otros); en este AVA se implementó un curso sobre Teoría de la Computación que se impartió a estudiantes de Ingeniería de la Computación en una Universidad del Japón. El autor utilizó el AVA como depositario de los materiales del curso, lecciones, sesiones síncronas y asíncronas, entre otras actividades. Posterior a la impartición del curso, realizó un análisis proveniente de la aplicación de una pretest y una postest al grupo que recibió esta capacitación, y se concluyó que el curso a través del AVA resultó ser efectivo para el aprendizaje. El autor sugirió realizar otros estudios que lleven a demostrar la efectividad de los AVA en cursos similares.

Una experiencia más sobre la capacitación a distancia, es la presentada por De la Cruz \& Gamboa (2005), quienes publicaron un estudio sobre la enseñanza de un curso de Inteligencia Artificial a estudiantes de Ingeniería, a través de un AVA. Este AVA permitió recibir capacitación y realizar prácticas de construcción de seres virtuales o 
agentes, que interactúan de manera independiente entre sí, en un ambiente controlado por computadora.

Estos autores encontraron que el curso a través del AVA contribuyó significativamente con el aprendizaje de los estudiantes que llevaron el curso de Inteligencia Artificial.

También Fernández (2003) presenta una experiencia de educación a distancia. Este autor desarrolló y evaluó un curso de capacitación en el área de la salud pública, utilizando ambientes virtuales de aprendizaje. Dicha evaluación concluyó que es factible el uso de ambientes de aprendizaje basados en la web. Además, el autor recomendó llevar a cabo investigaciones conducentes a evaluar la efectividad de este tipo de capacitación virtual.

\section{El Modelo de Evaluación de Acciones Formativas de Kirkpatrick}

Las evaluaciones de capacitación a través de e-learning, han demostrado ser eficientes y efectivas. Por ejemplo, Hall \& LeCavalier (2000) documentaron experiencias en IBM, que permitieron ofrecer cinco veces más aprendizaje, a un tercio del costo del método presencial, lo que produjo un ahorro de 200 millones de dólares. Otra experiencia documentada por los autores fue de la empresa Ernst \& Young, ellos minimizaron los costos de capacitación en un 35\%, al sustituir la capacitación presencial por un sistema de capacitación basado en AVA. Así mismo Tung. Huang, Keh \& Wai (2009) describen que la capacitación ofrecida a través de cursos a distancia, a militares de alto rango en Taiwan, alcanzó niveles altos de efectividad, debido a que los participantes demostraron las habilidades adquiridas, tiempo después de concluida la capacitación. También, Clark, Holstrom, \& Millaci (2009) reseñan algunos estudios que demostraron ser efectivos en cuanto al aprendizaje y aplicación del conocimiento y destrezas adquiridas. Finalmente, Brouse, McKnight, Basch \& LeBlanc (2010) señalan la efectividad de un curso sobre la Promoción y Cuidados de la Salud, impartido en un AVA.

La efectividad de los programas de capacitación se miden utilizando modelos de evaluación. Según Rodríguez (2005) un modelo clásico de evaluación de acciones normativas, utilizado por la industria y el sector académico, es el modelo que propuso Kirkpatrick en 1959. Este modelo propone la medición de las siguientes cuatro variables, para identificar la efectividad de un proceso de capacitación en modalidad presencial y a distancia: aprendizaje, satisfacción, aplicabilidad y resultados logrados (Kirkpatrick \& Kirkpatrick, 2006). De acuerdo a dichos autores las variables se definen de la siguiente manera:

1. El aprendizaje se refiere a los conocimientos que se obtuvieron, de acuerdo a los objetivos planteados.

2. La satisfacción involucra la percepción de los estudiantes con relación a la formación que recibieron.

3. La aplicación del conocimiento se refiere a la práctica de lo aprendido con relación a la formación que se recibió.

4. Los resultados logrados se relacionan con el producto que se alcanza, derivado de la aplicación del conocimiento en relación a la formación que se recibió. 


\section{Planteamiento del Problema}

Un Centro de Actualización y Capacitación del Magisterio (CACM) en una Entidad Federativa de México debe ofrecer capacitación a 17,232 docentes, que se encuentran asignados a diversos centros escolares, localizados en el espacio geográfico de esta Entidad (GEN, 2008). Tradicionalmente e invariablemente, desde su creación, este CACM ha ofertado capacitación únicamente en forma presencial. Esta forma de capacitación, ha generado en los últimos años un índice anual de capacitación menor al $10 \%$. Las razones de este bajo porcentaje de capacitación presencial se encuentran ligadas a razones presupuestarias y geográficas.

Estas limitantes se asocian al hecho de mantener, por parte del CACM, instalaciones físicas únicamente en la capital de la Entidad Federativa. Por lo que cada vez que se ofrece o planifica un curso de capacitación, necesariamente el CACM tiene que solventar los gastos de traslado, alimentación y hospedaje de aquellos docentes que viven lejos de la capital de la Entidad Federativa. Para el CACM, esto resulta costoso y en ocasiones imposible de cumplir. Consecuentemente, solo los docentes que radican en la capital de la Entidad Federativa, y en sus cercanías geográficas, son los beneficiarios del programa de capacitación del CACM.

Se infiere que las limitaciones presupuestarias y geográficas del CACM constituyen un notorio obstáculo que le dificulta cumplir con su objetivo. Evidentemente, los directivos del CACM tienen la necesidad de incrementar la cobertura de capacitación, y disminuir los requerimientos presupuestarios.

Por lo que, para intentar solventar esta necesidad, se propuso a las autoridades del CACM, experimentar con una modalidad de capacitación diferente a la que tradicionalmente venían ofreciendo: la educación a distancia. Dicho experimento consistió en ofrecer un curso del área de la computación en un Ambiente Virtual de Aprendizaje (AVA).

\section{Propósito de la Investigación}

El propósito de este estudio fue identificar la efectividad de un curso del área de la computación, ofrecido a través de un AVA, en un proceso de capacitación docente en el contexto de un CACM del Occidente de México.

\section{Preguntas de Investigación}

\section{Pregunta Principal}

¿Cuál es la efectividad que se alcanzó en el curso del área de la computación llevado a cabo en el AVA?

\section{Preguntas Especificas}

1. ¿Cuáles son las características de diseño instruccional que se consideraron para el curso del área de la computación que se implementó en el AVA? 
2. ¿Cuál es el aprendizaje que lograron los estudiantes que tomaron el curso sobre computación utilizando el AVA?

3. ¿Cuál es la satisfacción de los estudiantes que tomaron el curso del área de la computación utilizando el AVA?

4. ¿Cuál es la aplicabilidad de los conocimientos adquiridos que lograron los estudiantes que tomaron el curso sobre computación utilizando el AVA?

5. ¿Cuáles son los resultados que lograron los estudiantes que tomaron el curso sobre computación utilizando el AVA?

\section{Metodología}

El propósito de este estudio fue identificar la efectividad de un curso del área de la computación, ofrecido a través de un AVA. Para tal efecto, se elaboraron preguntas que proporcionaron una descripción cuantitativa de las variables. Las respuestas a dichas preguntas se buscaron implementando un enfoque de investigación cuantitativo, con diseño pre experimental, y un enfoque descriptivo, sin formulación de hipótesis. Este diseño fue seleccionado, ya que el mismo permitía un número de participantes reducido, no requería un proceso probabilístico para la constitución de la muestra y además, permitía realizar pretests y postests durante el periodo del estudio (Hernández, Fernández-Collado, \& Baptista, 2008).

\section{Participantes}

La población del estudio estuvo constituida por todos los docentes que se encuentran adscritos al CACM. Para la conformación de la muestra de estudio no se realizó proceso de selección probabilístico alguno, tampoco se distinguió género, antigüedad de servicio, ni edad del docente, entre otros. De acuerdo con Orellana (2009), un tamaño de clase de alrededor de 19 estudiantes, es percibido como el óptimo para obtener el mejor aprovechamiento de aprendizaje en un curso en línea; sin embargo para este experimento, por la probable deserción se consideró un tamaño de clase de 25 docentes. En consecuencia, la muestra fue de tipo intencional.

Como forma de homogenización de la muestra, sólo se inscribieron en el curso 25 docentes que acreditaron haber concluido el curso de Herramientas Básicas de la Computación, y que aseguraron no haber llevado previamente este curso o uno similar, dentro o fuera del ámbito del CACM. Estos docentes, constituyeron un único grupo de estudio, el cual fue la unidad de análisis. Desertaron 3 participantes por razones de salud y personales.

\section{Información Demográfica de los Participantes del Estudio}

El curso inició con 25 estudiantes, conformado por 14 personas del género femenino y 11 del género masculino. De los participantes que iniciaron el curso, 3 de ellos desertaron, lo que representa un 12\% de deserción (este porcentaje de deserción fue menor al estimado en la planeación del curso). Los motivos de la deserción fueron personales y de salud. 
Todos los participantes del estudio eran docentes, y se encuentran adscritos al nivel básico de educación (pre-escolar, primaria y secundaria) de la Secretaría de Educación Pública de una Entidad Federativa Mexicana. Todos ellos tienen estudios de licenciatura, destacando 11 que también cuentan con estudios de maestría. Así mismo, es importante mencionar que los docentes-estudiantes alcanzaron antigüedades laborales que oscilaron entre 2 y 24 años de servicio, lo que generó un promedio de antigüedad de 14,04 años, una moda de 15 años y una desviación estándar de 5,32 años.

Las edades biológica de los participantes del estudio variaron entre 22 y 44 años, promediando un valor de 34.04 años de edad, con una moda de 35 años y una desviación estándar de 5,32 años.

Los docentes-estudiantes provenían principalmente de escuelas secundarias técnicas, ubicadas en los diversos municipios de la Entidad Federativa.

\section{El Curso que se impartió}

El curso que se impartió, académicamente provenía del área de la computación. Los participantes tenían la necesidad de aprender sobre Microsoft Excel (ME) 2007, por tanto se realizó un diseño instruccional de éste para colocarlo en la plataforma Moodle. Para su diseño instruccional se tomaron en cuenta los siguientes elementos: el instructor, el estudiante, el contenido, el método, el ambiente, el material, las estrategias didácticas, la tecnología y el proceso de evaluación (Simonson et al., 2006).

Con estas características y elementos de diseño de instrucción, se buscó el modelo más apropiado para el curso del área de computación en el AVA que se impartió. Se encontraron y evaluaron varios modelos, algunos de ellos fueron los siguientes: modelo Dorrego \& García; modelo ASSURE; y modelo Dick, Carey \& Carey.

De estos modelos, se eligió el modelo Dick, Carey \& Carey (2009), porque satisfizo las características y elementos de diseño instruccional propuestos por Simonson et al. (2006). La etapas del modelo que se aplicaron son: identificación de las metas, identificación de objetivos, examen de habilidades, estrategias y materiales instruccionales, evaluación, evaluación y revisión de la instrucción. La aplicación de este modelo, produjo el diseño instruccional del curso que se impartió a los participantes del estudio durante tres meses.

\section{Instrumentos}

Durante el estudio, se requirió del uso de diferentes instrumentos de recolección de datos. Por ejemplo, para medir el aprendizaje en el experimento, se utilizó una pretest y una postest. Para medir la satisfacción, la aplicabilidad del conocimiento adquirido y los resultados logrados por los participantes en el experimento, se llevó a cabo una recolección de datos, utilizando como instrumentos cuestionarios electrónicos de preguntas cerradas pre-codificadas, con respuestas de tipo ordinal, que emplearon el escalamiento de Likert. 


\section{Validez de los Instrumentos Aplicados}

La pretest (Apéndice A), la postest (Apéndice B); y los cuestionarios utilizados para medir la satisfacción (Apéndice $\mathrm{C}$ ), la aplicación del conocimiento adquirido (Apéndice D), y los resultados logrados (Apéndice E); demostraron contar con validez de contenido y validez de criterio; esto de acuerdo a la descripción que se presenta en la sección de Instrumentos.

\section{Confiabilidad de los Instrumentos Aplicados}

La pretest (Apéndice A) y la postest (Apéndice B), fueron sometidos al procedimiento de confiabilidad denominado Medida de Estabilidad (confiabilidad por testretest); y de acuerdo a lo señalado en la sección de Instrumentos, éstas fueron encontradas confiables para su aplicación.

La confiabilidad de los cuestionarios electrónicos que se aplicaron para medir la satisfacción (Apéndice C), la aplicabilidad de conocimientos (Apéndice D), y los resultados logrados (Apéndice E); fueron sometidos al Método de Mitades Partidas (splithalves). De acuerdo a lo destacado en la sección de Instrumentos, se encontró que estos instrumentos eran confiables, y que se encontraban preparados para su aplicación.

Además de la confiabilidad mencionada, después de la recolección de datos sobre la satisfacción, aplicación del conocimiento adquirido y resultados logrados; se realizó la prueba de confiabilidad denominada consistencia interna; calculándose el estadístico conocido como coeficiente Alfa Cronbach. Para todos los casos, este coeficiente resulto mayor a 0.70 , por lo que según Litwin (2003) pueden ser considerados como indicadores de confiabilidad aceptable. Así que con la confianza de estos coeficientes, se procedió al análisis respectivo.

\section{Ponderación de Reactivos y Cálculo de Porcentajes}

La ponderación de las respuestas de los reactivos de los cuestionarios de satisfacción, aplicación del conocimiento y resultados logrados; se llevó a cabo de la siguiente manera: un valor de 5 para respuestas Muy de Acuerdo (Muy Satisfecho), un valor de 4 para respuestas De Acuerdo (Satisfecho), un valor de 3 para respuestas Neutral (Regularmente Satisfecho), un valor de 2 para respuestas En Desacuerdo (Insatisfecho), y un valor de 1 para Muy en Desacuerdo (Muy Insatisfecho).

Después de realizar la ponderación de reactivos, se calculó un puntaje consolidado que representó los porcentajes de satisfacción, conocimientos adquiridos, y resultados logrados para cada uno de los aprendices. El cálculo de dicho puntaje consolidado se realizó de acuerdo al procedimiento basado en promedios, propuesto por Litwin (2003, p. 48). Según este autor, este procedimiento es utilizado en estudios donde todas las preguntas del cuestionario, tienen el mismo rango de valores en sus respuestas. El procedimiento propuesto por Litwin (2003) que se aplicó para cada uno de los cuestionarios fue el siguiente:

1. La respuesta señalada por el estudiante, en cada una de las preguntas, se dividió entre 5 (valor máximo posible). 
2. Con los valores obtenidos en el punto anterior (procedentes de la división efectuada en cada una de las respuestas del cuestionario), se realizó una sumatoria.

3. La sumatoria del punto anterior se dividió entre el total de preguntas del cuestionario.

4. Finalmente, el resultado de la división, que se menciona en el punto anterior, se multiplicó por 100.

Así se obtuvo el porcentaje correspondiente para cada cuestionario aplicado.

\section{Procedimiento}

Para el logro de los objetivos de este estudio, se realizaron diversas actividades procedimentales que proporcionaron respuestas a las preguntas planteadas. Para identificar las características del diseño instruccional del curso del área de la computación que se implementó en el AVA, se realizaron búsquedas en bases de datos electrónicas, revistas arbitradas y libros de textos. Posteriormente se realizó el diseño del curso de ME 2007 para un AVA, teniendo en consideración las características de diseño instruccional encontradas y las etapas propuestas por Dick, Carey \& Carey (2009).

Realizado el diseño instruccional del curso, se inició el proceso de búsqueda y localización de los instrumentos de recolección de datos. Localizados los instrumentos de recolección de datos, se analizaron y evaluaron diversas plataformas virtuales de aprendizaje. Este proceso de análisis y evaluación determinó que la plataforma virtual de aprendizaje más apropiada para el presupuesto del CACM, era Moodle. Por lo que se seleccionó esta plataforma virtual de aprendizaje, sobre la cual se instaló el curso de ME 2007 (materiales instruccionales, foros de discusión, sesiones síncronas, chats, asignaciones individuales y en grupo, espacios para preguntas elaboradas por el aprendiz y su respectiva retroalimentación, buzón para comentarios, sugerencias al instructor, entre otros), y los instrumentos de recolección de datos. Instalado el curso, se realizaron pruebas de usabilidad de la plataforma Moodle.

A continuación se llevó a cabo la conformación de la muestra de estudio. Posteriormente, se inició la impartición del curso. Como primera actividad del curso se realizó la aplicación de la pretest, y como última actividad la aplicación de la postest. Estas pruebas permitieron recolectar datos para medir el aprendizaje.

Los datos recolectados de la pretest y la postest se sometieron al cálculo de una prueba paramétrica t-student, esto con el propósito de comprobar las diferencias estadísticas significativas entre la primera y la segunda medida. El uso del estadístico tstudent con una muestra pequeña se debió a que el grupo de participantes fue proporcionado por las autoridades para esta investigación. Es decir, fue una muestra intencional-dirigida. Por otro lado, y posterior a los cálculos que determinaron el aprendizaje, se procedió a recolectar información para obtener datos sobre la satisfacción. Para esto, al finalizar el curso, en la plataforma Moodle se colocó a disposición de los aprendices, un cuestionario electrónico para medir la satisfacción. 
Pasado un mes de concluida la capacitación, y con el propósito de obtener datos sobre la aplicación de los conocimientos adquiridos, se envió electrónicamente a los aprendices, el cuestionario respectivo. Al igual que con el cuestionario sobre la aplicación de los conocimientos adquiridos, pasado un mes de concluida la capacitación, e intentando obtener datos sobre los resultados logrados, se envió electrónicamente a los directivos de las escuelas, el cuestionario correspondiente.

Una vez que se recolectaron los datos sobre la satisfacción, aplicación del conocimiento y resultados logrados, se calculó el puntaje consolidado de cada variable, y se determinó que habría satisfacción, aplicación del conocimiento, y resultados logrados si la media aritmética del valor de cada variable llegaba a ser $\geq 60 \%$. Finalmente, se realizó el análisis de datos y presentación de resultados.

\section{Resultados}

La presentación de los resultados de un estudio son parte medular de un proceso de investigación (Fink, 2003). Estos resultados crean una conexión entre el público destino, la investigación y el investigador (Bourque, 2003). Por esto, la forma en cómo se presentan los resultados determina en gran medida el impacto e importancia que el público destino perciba de ellos (Fink, 2003). Dicho autor argumenta que los resultados de una investigación pueden ser presentados en alguna de las siguientes formas: a) descriptiva, b) de relación, c) de comparación, o d) de predicción.

Los resultados de este estudio de investigación se presentan en forma descriptiva y comparativa, y provienen de tres fuentes de datos: a) datos demográficos de los participantes, b) información sobre el diseño instruccional del curso en el AVA, y c) datos recolectados a través de los diferentes instrumentos que se aplicaron. Una de las preguntas se respondió, a partir de la información que se encontró sobre el diseño instruccional para cursos de capacitación en un AVA. El resto de las preguntas se respondieron con datos provenientes de los diferentes instrumentos de recolección de datos aplicados.

Los datos demográficos de los participantes del estudio; la información sobre el diseño de cursos de capacitación en un AVA; al igual que los resultados para indicar la satisfacción, la aplicación del conocimiento adquirido y los resultados logrados; se presentan en forma descriptiva. Únicamente los resultados sobre el aprendizaje se presentan en forma comparativa. Los resultados se presentan en forma individual, lo cual se facilitó debido al número reducido de participantes.

\section{Resultados sobre el Aprendizaje Alcanzado}

Como parte del proceso llevado a cabo para medir el aprendizaje por los participantes del curso de computación en el AVA, se aplicó una pretest y una postest. La pretest se aplicó a 25 aprendices. En la recolección de datos de la pretest se destaca que el porcentaje de calificaciones más bajo fue de $30 \%$, mientras que el más alto fue de $70 \%$ (considerando una escala de 0 a 100\%). El promedio de las calificaciones fue de 
$46,59 \%$, mientras que la moda fue de 40 , la mediana de 47,5, y la desviación estándar de 10,16 .

Por otro lado, la postest fue aplicada a 22 participantes (3 participantes desertaron) una vez finalizado el curso. Los datos recolectados indican que el porcentaje de calificaciones más bajo fue de $85 \%$, mientras que el más alto fue de $100 \%$ (considerando una escala de 0 a $100 \%$ ). El promedio de calificaciones alcanzado en la pos prueba fue de $91,59 \%$, mientras que la moda fue de 90 , la mediana de 90 , y la desviación estándar de 5,85 .

Empleando los datos obtenidos en ambas pruebas, y con la intención de hacer un análisis comparativo, se presentan en la Tabla 1 las diferencias porcentuales de cada uno de los participantes en ambas pruebas. En ésta se aprecia que existen diferencias porcentuales entre las calificaciones logradas en la pretest y la postest. Para todos los casos las calificaciones en la postest fueron mayores que las calificaciones de la pretest. La menor diferencia porcentual entre la pretest y la postest fue de $30 \%$, para los estudiantes 1 y 2 ; mientras que la mayor diferencia fue de $60 \%$, para los estudiantes 9 y 22. Las diferencias porcentuales encontradas permitieron calcular el promedio de diferencias ( $\mathrm{pd}=45 \%)$, y la desviación estándar $(\mathrm{sd}=9,128)$.

\begin{tabular}{cccc}
\hline Participante & Pretest $(\%)$ & Postest $(\%)$ & Diferencia Porcentual \\
\hline 1 & 60 & 90 & 30 \\
2 & 70 & 100 & 30 \\
3 & 45 & 95 & 50 \\
4 & 50 & 85 & 35 \\
5 & 55 & 90 & 35 \\
6 & 40 & 85 & 45 \\
7 & 50 & 90 & 40 \\
8 & 40 & 95 & 55 \\
9 & 30 & 90 & 60 \\
10 & 55 & 100 & 45 \\
11 & 50 & 95 & 45 \\
12 & 40 & 85 & 45 \\
13 & 30 & 80 & 50 \\
14 & 50 & 90 & 40 \\
15 & 40 & 95 & 55 \\
16 & 60 & 95 & 35 \\
17 & 55 & 100 & 45 \\
18 & 40 & 95 & 55 \\
19 & 50 & 85 & 35 \\
20 & 35 & 85 & 50 \\
21 & 40 & 90 & 50 \\
22 & 40 & 100 & 60 \\
\hline
\end{tabular}

Tabla 1. Diferencias porcentuales de las calificaciones obtenidas en la pretest y la postest

Las diferencias porcentuales presentadas en la Tabla 1, para cada uno de los participantes, se pueden apreciar gráficamente en la Figura 1. En dicha Figura se observa que la pretest y la postest presentan altos y bajos en sus curvas, manteniéndose en todos los puntos, la curva de la postest por arriba de la curva de la pretest. Lo anteriormente ex- 
puesto demuestra gráficamente las diferencias entre los resultados obtenidos en ambas pruebas.

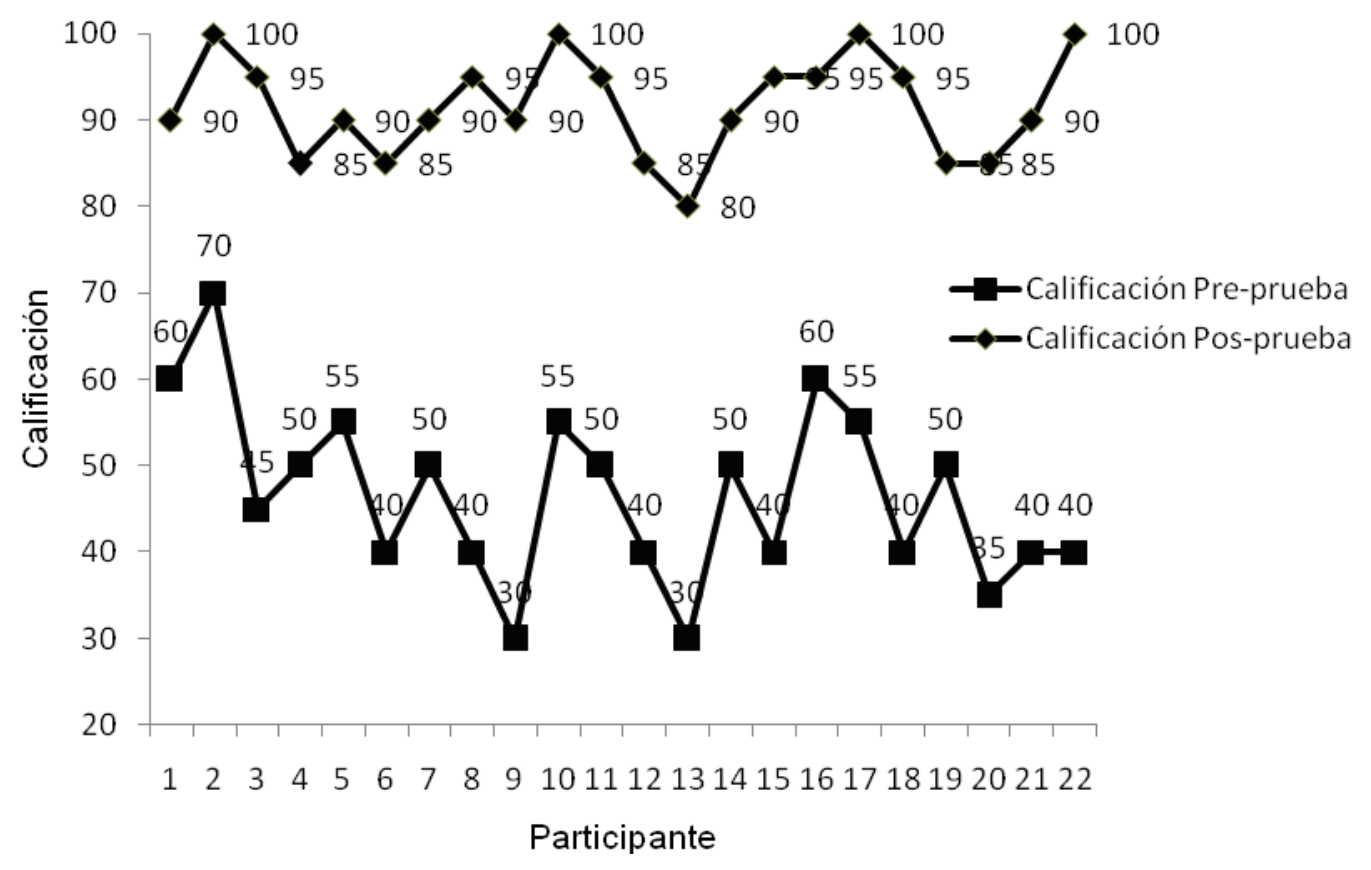

Figura 1. Diferencias porcentuales entre las calificaciones obtenidas por cada estudiante en la pretest y la postest.

La prueba $\mathrm{t}$ de Student para muestras relacionadas resultó significativa $(\mathrm{t}=23,12, \mathrm{p}$ $=0.000$ ) con un $\alpha$ de 0,05 . Esto nos permite concluir que las calificaciones de los estudiantes fueron superiores en el postest, por lo que existieron diferencias significativas en el aprendizaje.

\section{Resultados sobre la Satisfacción Alcanzada}

Los datos recolectados por el instrumento diseñado para medir la satisfacción fueron sometidos a la prueba de consistencia interna, es decir se calculó el coeficiente Alfa Cronbach, lográndose un valor de 0,781. Este valor estadístico del Alfa Cronbach, según Litwin (2003) es aceptable, lo que indica que los datos son confiables, y que pueden utilizarse para presentar resultados.

Con los resultados de cada uno de los reactivos del cuestionario se identificaron los valores mínimos y máximos, además se calculó la media, la desviación estándar y la varianza. En la Tabla 2 se puede observar que el valor mínimo se dio para el reactivo de duración del curso, lo que indica que algunos estudiantes que estuvieron regularmente satisfechos con la duración del curso. Entre tanto, también se aprecia que la máxima calificación para los reactivos fue de 5 , lo que implica que algunos estudiantes 
estuvieron muy satisfechos, en todos los rubros. Los valores medios para todos los reactivos, oscilan desde un valor de 4,77 hasta 4,91, todos ellos muy similares, lo que se traduce que en promedio se tuvieron estudiantes muy satisfechos (un valor cercano al 5). Se puede apreciar también, que las columnas de medida de dispersión y varianza, contienen valores bajos, menores a 1 , ya que los valores medios para todos los reactivos fueron muy similares.

\begin{tabular}{lccccccc}
\hline Satisfecho con... & $N$ & Min & Max & $\bar{X}$ & S & $\sigma$ X & Var \\
\hline Organización del curso & 22 & 4 & 5 & 4,91 & 0,294 & 0,0626 & 0,087 \\
Contenido del curso & 22 & 4 & 5 & 4,82 & 0,395 & 0,0842 & 0,156 \\
Duración del curso & 22 & 3 & 5 & 4,36 & 0,658 & 0,1402 & 0,433 \\
Materiales instruccionales & 22 & 4 & 5 & 4,82 & 0,395 & 0,0842 & 0,156 \\
Actividades académicas & 22 & 4 & 5 & 4,86 & 0,351 & 0,0748 & 0,123 \\
Conocimientos y habilidad adquiridos & 22 & 4 & 5 & 4,77 & 0,429 & 0,0414 & 0,184 \\
Disponibilidad y atención del instructor & 22 & 4 & 5 & 4,82 & 0,395 & 0,0842 & 0,156 \\
Retroalimentación del instructor & 22 & 4 & 5 & 4,86 & 0,351 & 0,0748 & 0,123 \\
Expectativas de aprendizaje & 22 & 4 & 5 & 4,86 & 0,351 & 0,0748 & 0,123 \\
Experiencia de aprendizaje & 22 & 4 & 5 & 4,86 & 0,351 & 0,0748 & 0,123 \\
\hline
\end{tabular}

Tabla 2. Estadísticos descriptivos por reactivo del cuestionario de satisfacción.

Nota. $N=$ número de reactivos evaluados; Min = Valor mínimo encontrado en reactivo; Max $=$ Valor máximo encontrado en reactivo; $\bar{X}=$ Valor medio calculado en el reactivo; $\mathrm{S}=$ Desviación Estándar; Var = Varianza; y $\sigma_{x}=$ Error típico.

Con los datos de la Tabla 2 se calcularon los porcentajes de satisfacción de cada uno de los participantes (siguiendo el procedimiento descrito al inicio de esta sección de resultados). Estos valores ocurrieron desde un valor mínimo de $90 \%$ hasta un valor máximo de $100 \%$. La mayoría de los estudiantes lograron valores de satisfacción superiores al 95\%. El promedio de satisfacción grupal fue de 95,9\%, la mediana de 96, y la moda de 100. Utilizando estos datos se generó la Tabla 3. En dicha Tabla se puede observar que 7 estudiantes mostraron la mayor frecuencia de satisfacción, los cuales manifestaron estar $100 \%$ satisfechos. Entre tanto, las menores frecuencias de satisfacción la mostraron 3 estudiantes, quienes manifestaron estar satisfechos en un 90\%. En general, se puede apreciar en la Tabla 3, que las frecuencias de los porcentajes de satisfacción, se encuentran por arriba del 90\% de satisfacción, y en 7 casos llegan hasta el $100 \%$.

\begin{tabular}{cc}
\hline Satisfacción (\%) & Frecuencias \\
\hline 90 & 3 \\
92 & 4 \\
94 & 0 \\
96 & 6 \\
98 & 2 \\
100 & 7 \\
\hline
\end{tabular}

Tabla 3. Frecuencias de los porcentajes de satisfacción

\section{Resultados sobre la Aplicación del Conocimiento Adquirido}

Los datos del cuestionario electrónico sobre la aplicación de conocimientos adquirido fueron sometidos a la prueba de consistencia interna, se calculó el coeficiente Alfa Cronbach lográndose un valor de 0,734. El valor estadístico Alfa Cronbach obtenido, 
según Litwin (2003), es aceptable, lo que indica que los datos son confiables, y que pueden utilizarse para presentar resultados.

Con los resultados de cada uno de los reactivos del cuestionario se identificaron los valores mínimos y máximos, además se calculó la media, la desviación estándar y la varianza. En la Tabla 4 se presentan algunos estadísticos descriptivos, entre ellos los valores mínimos, máximos, media, desviación estándar y varianza.

\begin{tabular}{lccccccc}
\hline Los conocimientos adquiridos... & $N$ & Min & Max & $\bar{X}$ & S & $\sigma$ X & Var \\
\hline Se aplicaron en su ámbito laboral & 22 & 3 & 5 & 3,95 & 0,950 & 0,2025 & 0,903 \\
Mejoraron el desempeño de funcione & 22 & 3 & 5 & 4,09 & 0,811 & 0,1729 & 0,658 \\
Permitió nuevas formas de trabajo & 22 & 3 & 5 & 4,18 & 0,795 & 0,1654 & 0,632 \\
Incrementaron su motivación & 22 & 3 & 5 & 4,00 & 0,873 & 0,2132 & 0,762 \\
Mejoraron su desarrollo personal & 22 & 3 & 5 & 3,86 & 0,774 & 0,1650 & 0,600 \\
Permitió desarrollo nueva habilidad & 22 & 3 & 5 & 4,23 & 0,752 & 0,1603 & 0,565 \\
Permitió mejorar actitud & 22 & 3 & 5 & 4,23 & 0,752 & 0,1603 & 0,565 \\
\hline
\end{tabular}

Tabla 4. Estadísticos descriptivos por reactivo del cuestionario de aplicación del conocimiento adquirido

Nota $. N=$ número de reactivos evaluados; Min = Valor mínimo encontrado en reactivo; Max $=$ Valor máximo encontrado en reactivo; $\bar{X}=$ Valor medio calculado en el reactivo; $\mathrm{S}=$ Desviación Estándar; Var $=$ Varianza; y $\sigma_{x}=$ Error típico.

Se puede observar en la Tabla 4, que el valor mínimo en la columna Min fue de 3, lo que indica que algunos estudiantes estuvieron regularmente de acuerdo con la aplicación de sus conocimientos. Entre tanto, también, se aprecia que la máxima calificación para los reactivos fue de 5, lo que implica que algunos estudiantes estuvieron muy de acuerdo con la aplicación de sus conocimientos. Los valores medios para todos los reactivos van desde 3,86 hasta 4,23 , todos ellos muy similares, lo que quiere decir que en promedio los estudiantes estuvieron de acuerdo con la aplicación de sus conocimientos (valor de 4). Se puede apreciar también que las columnas de medida de dispersión (DE) y varianza, contienen valores relativamente bajos, menores a 1, esto debido a que los valores medios para todos los reactivos fueron muy similares. Es conveniente destacar que la Tabla 4 no muestra valores de 2 (en desacuerdo) ni de1 (muy en desacuerdo) para las columnas de los valores mínimos y máximos. Lo anterior podría ser un indicativo de que los participantes del curso, aplicaron los conocimientos adquiridos.

Adicionalmente se calculó el promedio de aplicación del conocimiento grupal que fue de $81,18 \%$, resultando una mediana de 81 , y una moda de 80 . Utilizando los las datos obtenidos se presenta la Tabla 5, la cual muestra los rangos de calificaciones con sus respectivas frecuencias. Los datos de dicha Tabla señalan que 20 estudiantes se encuentran ubicados en los rangos 71-80 y 81-90, lo que representa un 91,6\%, mientras que 2 estudiantes se encuentran fuera de estos rangos, uno en el rango de 91-100 y el otro en el rango de 60-70.

\begin{tabular}{rc}
\hline Rango & Frecuencias \\
\hline $60-70$ & 1 \\
$71-80$ & 10 \\
$81-90$ & 10 \\
$91-100$ & 1 \\
\hline
\end{tabular}

Tabla 5. Frecuencias de los porcentajes de aplicación del conocimiento adquirido 


\section{Resultados Logrados}

El cuestionario electrónico para la recolección de datos sobre los resultados logrados, se envió a 22 directivos de los centros educativos de procedencia de los participantes que conformaron la muestra. Este cuestionario alcanzó un nivel de respuesta de $86,3 \%$. Los datos recolectados fueron sometidos a una prueba de consistencia interna, es decir se calculó el coeficiente Alfa Cronbach lográndose un valor de 0,718. El valor estadístico Alfa Cronbach obtenido, según Litwin (2003), es aceptable, lo que indica que los datos son confiables, y pueden utilizarse para presentar resultados.

Por consiguiente, la Tabla 6 presenta algunos estadísticos descriptivos, entre ellos los valores mínimos, máximos, media, desviación estándar y varianza.

\begin{tabular}{lccccccc}
\hline Reactivos & $N$ & Min & Max & $\bar{x}$ & $\mathrm{~S}$ & $\sigma_{\mathrm{x}}$ & Var \\
\hline Se obtuvieron de su ámbito laboral & 22 & 3 & 5 & 4.16 & 0,834 & 0,177 & 0,696 \\
Mejoraron el desempeño de funciones & 22 & 3 & 5 & 4.21 & 0,855 & 0,182 & 0,731 \\
Permitieron nuevas formas de trabajo & 22 & 3 & 5 & 3.89 & 0,809 & 0,172 & 0,655 \\
Incrementaron su motivación & 22 & 3 & 5 & 4.11 & 0,809 & 0,172 & 0,655 \\
Mejoraron su desarrollo personal & 22 & 3 & 5 & 3.89 & 0,809 & 0,172 & 0,655 \\
Permitieron el desarrollo de nuevas habili- & 22 & 3 & 5 & 3.84 & 0,834 & 0,177 & 0,696 \\
dades & 22 & 3 & 5 & 3.47 & 0,697 & 0,148 & 0,485 \\
Permitieron mejorar su actitud & 22 &
\end{tabular}

Tabla 6. Estadísticos descriptivos por reactivo del cuestionario de resultados obtenidos

Nota. $N$ = número de reactivos evaluados; Min = Valor mínimo encontrado en reactivo; $\mathrm{Max}=$ Valor máximo encontrado en reactivo; $\bar{x}=$ Valor medio calculado en el reactivo; $\mathrm{S}=$ Desviación Estándar; Var $=$ Varianza; y $\sigma_{\mathrm{x}}=$ Error típico

Se puede observar en la Tabla 6, que el valor mínimo fue 3 (regularmente de acuerdo) y el valor máximo fue de 5 (muy de acuerdo), lo que indica que algunos directivos estuvieron regularmente de acuerdo, de acuerdo y muy de acuerdo, con los resultados logrados por el estudiante que recibió el curso. Los valores medios para todos los reactivos, oscilan entre 3,47 y 4,21, lo que quiere decir que se alcanzaron los resultados esperados, por parte de los directivos de las escuelas. Se puede apreciar también, que las columnas de medida de dispersión (DE) y varianza, contienen valores relativamente bajos, menores a 1, esto debido a que los valores medios para todos los reactivos fueron muy similares. Es conveniente destacar que la Tabla no muestra valores de 2 (en desacuerdo) ni de1 (muy en desacuerdo) para las columnas de valores mínimos y máximos.

Los resultados logrados, según el cuestionario aplicado a los directivos, oscilan entre el $68 \%$ y el $91 \%$. El directivo que menos estuvo de acuerdo con los resultados logrados por su colaborar fue el 3, mientras que el directivo que más estuvo de acuerdo, con los resultados logrados fue el 16.

\begin{tabular}{cc}
\hline Resultados obtenidos (\%) & Frecuencias \\
\hline $60-70$ & 1 \\
$71-80$ & 11 \\
$81-90$ & 6 \\
$91-100$ & 1 \\
\hline
\end{tabular}

Tabla 7. Frecuencias de los porcentajes de resultados obtenidos 
La Tabla 7 muestra que la mayoría de los directivos estuvieron de acuerdo en un rango del 70 al $90 \%$ con los resultados logrados por su colaborador. Solo un directivo (el 3) se mostró de acuerdo en un valor por abajo del 70\%, y un directivo (el 16) se mostró de acuerdo en un valor por arriba del $90 \%$.

\section{Discusión y Conclusiones}

\section{Interpretación de los Resultados}

A. Acerca del aprendizaje logrado. Se encontraron diferencias en el aprendizaje de los estudiantes que participaron en el curso experimental. El análisis de las calificaciones en la pretest y la postest, a través del estadístico t-student pareado; permitió determinar que el curso promovió el aprendizaje entre los participantes. Aparentemente, la instrucción proporcionada mediante un AVA, fue efectiva para aprender el contenido de ME 2007. Por tal motivo, se concluyó que la instrucción a través del AVA, fue efectiva para lograr el aprendizaje de ME 2007, en un proceso de capacitación docente.

Estos resultados son consistentes con lo señalado por Hamada (2007) quien implementó un curso sobre Teoría de la Computación en un AVA, y concluyó que dicho curso generó aprendizaje entre los participantes. Otros estudios también han avalado el aprendizaje en los AVAs, por ejemplo el de De la Cruz \& Gamboa (2005) quienes impartieron un curso sobre Inteligencia Artificial en un AVA, aplicaron una pretest y postest a los participantes, y determinaron que el curso promovió el aprendizaje entre los estudiantes.

B. Acerca de la satisfacción lograda. Se encontraron porcentajes de satisfacción estudiantil mayores o iguales al $90 \%$, por parte de los participantes del curso experimental. Estos porcentajes de satisfacción individual generaron un promedio de satisfacción de 95,9\%. El análisis de estos resultados de satisfacción, permitió determinar que los estudiantes estuvieron satisfechos con el curso. Aparentemente, el proceso de instrucción a través del AVA, fue efectivo para generar satisfacción entre los estudiantes. Por tal motivo, se concluyó que la instrucción a través del AVA, fue satisfactoria para los participantes, en su proceso de capacitación docente.

Estos resultados son comparables con los de Glass \& Sue (2008) quienes reportaron una satisfacción estudiantil cercana al $90 \%$. Otro estudio, como el de Richardson \& Swan (2003) también demostró que los estudiantes universitarios, participantes de cursos ofertados en ambientes virtuales, reportaron altos niveles de satisfacción en este tipo de instrucción.

C. Acerca de la aplicabilidad de los conocimientos adquiridos. Se encontraron porcentajes de aplicación del conocimiento adquirido mayores o iguales al $65 \%$, por parte de los participantes del curso experimental. Estos porcentajes individuales de aplicación del conocimiento adquirido, generaron un promedio de $81,18 \%$. El análisis de estos de estos resultados, permitió determinar que los estudiantes 
aplicaron el conocimiento adquirido. Aparentemente, el proceso de instrucción a través del AVA, fue efectivo para ayudar a los estudiantes a aplicar el conocimiento adquirido. Por tal motivo, se concluyó que la instrucción ofrecida sobre ME 2007 a través del AVA, fue efectiva para promover la aplicación de los conocimientos adquiridos, por parte de los participantes.

Estos resultados son similares a los destacados por Sendag \& Odabasi (2009), quienes encontraron que los conocimientos adquiridos por un grupo de profesores, en un curso a distancia, fueron aplicados para resolver problemas en línea, en su entorno laboral. Otros estudios también han avalado la aplicación de los conocimientos por parte de los participantes en procesos de capacitación a distancia, por ejemplo Hall \& LeCavalier (2000), encontraron que algunas empresas, como Ernest \& Young, han manifestado que sus trabajadores aplican los conocimientos adquiridos, después de un proceso de capacitación virtual.

D. Acerca de los resultados logrados. Se encontraron porcentajes de resultados logrados mayores o iguales al 68\%, por parte de los participantes del curso experimental. Estos porcentajes individuales de resultados logrados, generaron un promedio de $78,5 \%$. El análisis de estos resultados permitió determinar que los estudiantes lograron los resultados esperados por sus directivos. Aparentemente, el proceso de instrucción a través del AVA, fue efectivo para lograr los resultados esperados. Por tal motivo, se concluyó que la instrucción ofrecida sobre ME 2007 a través del AVA, fue efectiva para lograr los resultados esperados, según la opinión de los directivos de los participantes.

Estos resultados son equiparables con los señalados por Strother (2002), quien destaca que directivos de organismos corporativos y académicos, han encontrado que se lograron los resultados esperados, después de un proceso de capacitación virtual. Otro estudio, realizado por Hall \& LeCavalier (2000) también indicó que empresas como IBM, han manifestado su satisfacción por los resultados logrados por sus colaboradores, después de procesos de capacitación en línea.

E. Acerca de la efectividad en el curso llevado a cabo en el AVA. Se encontró que los participantes aprendieron en el curso de capacitación ofrecido en el AVA. Además, se sintieron satisfechos; aplicaron los conocimientos adquiridos; y se alcanzaron los resultados esperados, según sus directivos. El análisis de estos resultados, de acuerdo al modelo de acciones formativas de Donald Kirkpatrick, permitió determinar que el curso sobre ME 2007 en el AVA, fue efectivo. Aparentemente, la instrucción proporcionada mediante un AVA, fue efectiva. Por tal motivo, se concluyó que la instrucción ofrecida a los docentes del CACM, sobre ME 2007 a través del AVA, fue efectiva.

La efectividad encontrada en este estudio, es coincidente con lo que señalan en sus investigaciones autores tales como Grisolía \& Corral (2005); Hamada (2007); De la Cruz \& Gamboa (2005); Fernández (2003); Tung et al. (2009); Clark et al. (2009); y Brouse et al. (2010). 


\section{Referencias bibliográficas}

ALMANZOR, C. (2007). Importancia de la capacitación pedagógica del docente. Disponible en http://www.uag.mx/63/a04-02.htm

BOURQUE, L. B. (2003). How to conduct self-administered and mail surveys (2a. ed.). London, England: Sage.

BROUSE, C. H., McKnight, K. R., Basch, C. E. \& LeBlanc, M. (2010). A Pilot Study of Instructor Factors and Student Preferences. Journal of Educational Technology Systems, 1(38), 51-62.

CHEN, J. C. \& SEONG, C. T. (2005). A feasible constructivist instructional development model for virtual reality. Educational Technology Research and Development, 53(1), 111-123.

CLARK, M., HOLSTROM, L. \& MILLACI, A. M. (2009). University of Cincinnati: Case study of online student success. Journal of Asynchronous Learning Networks, 3(13), 49-55.

DE LA CRUZ, G. \& GAMBOA, F. (2005). Exploración del aprendizaje de los estudiantes haciendo uso de ambientes colaborativos: Enseñando inteligencia artificial. Disponible en http://www.utpl.edu.ec/ried/images/pdfs/vol8-1-2/exploracion_aprendizaje.pdf

DICK, W., CAREY, L. \& CAREY, J. (2009). The systematic design of instruction (7th ed.). New York, Ny, E.E.U.U.: Allyn \& Bacon.

DORREGO, E. \& GARCÍA, A. M. (1991). Dos modelos para la producción y evaluación de modelos instruccionales. Caracas Venezuela: Universidad Central de Venezuela, Facultad de Humanidades y Educación.

FERNÁNDEZ, M. (2003). Desarrollo de un ambiente virtual de aprendizaje en salud pública. Disertación doctoral no publicada, Nova Southeastern University, Fort Lauderdale, Florida, EE. UU.

FINK, A. (2003). The survey handbook (2a. ed.). London, England: Sage.

GEN. (2008). Gobierno del Estado de Nayarit. Disponible en http:/www.nayarit.gob.mx/

GLASS, J. \& SUE, V. (2008). Students' preferences, satisfaction, and perceived learning in an online mathematics class. MERLOT Journal of Online Learning and Teaching, 4(3), 325-338.

GRISOLÍA, C. M. \& CORRAL, M. C. (2005). Experiencia de capacitación virtual. Disponible en http://salvador.edu.ar

HALL, B. \& LECAVALIER, J. (2000). E-learning across the enterprise: The benchmarking study of best practices. Sunnyvale, CA, E. E. U. U: Brandon-Hall research.

HAMADA, M (2007). An integrated virtual environment. Technology for E-learning and Digital Entertainment, 12(2), 422-432.

HERNÁNDEZ, R., FERNÁNDEZ-COLLADO, C. \& BAPTISTA, P. (2008). Metodología de la investigación (4a. ed.). Cd. de México, D. F., México: McGraw-Hill Interamericana.

KIRKPATRICK, D. L. \& KIRKPATRICK, J. D. (2006). Evaluating training programs: The four levels (3a. ed.). San Francisco, CA, E.E.U.U. : Berrett-Koehler. 
LITWIN, M. S. (2003). How to assess and interpret survey psychometrics (2a. ed.). London, England: Sage.

MENDOZA, J. (2003). e-learning, el futuro de la educación a distancia. Disponible en http:/www.informaticamilenium.com.mx/Paginas/mn/ articulo78.htm

NEW YORK STATE EDUCATION DEPARTMENT (2007). Principles and operational criteria for good practice in distance higher education. Disponible en http://www.highered.nysed.gov/ocue/ded/practice.html

ORELLANA, A. (2009). Class size and interaction in online courses. En A. Orellana, T. L. Hudgins \& M. Simonson (Eds.), The perfect online course: Best practices for designing and teaching (pp. 117-135). Charlotte, NC, E.E.U.U.: Information Age Publishing.

RICHARDSON, J. \& SWAN, K (2003). Examining social presence in online courses in relation to students' perceived learning and satisfaction. JALN, 7(1), 68-88.

RODRÍGUEZ, J. (2005). El modelo Kirkpatrick para la evaluación de la formación. Disponible en http://www.uhu.es/yolanda.pelayo/docencia/Virtualizacion/2contenidos/parte\%204/MODELO\%20DE\%20KIRCKPATRICK.pdf

SENDAG, S. \& ODABASI, H. F. (2009). Effects of an online problem based learning course on content knowledge acquisition and critical thinking skills. Computers $\&$ Education, 53(1), 132-141.

SEÑAS, P. (2005). Aprendizaje basado en la web. Disponible en http://cs.uns.edu.ar/jeitics2005/Trabajos/pdf/51.pdf

SIMONSON, M., SMALDINO, S., ALBRIGHT, M., \& ZVACEK, S. (2006). Teaching and learning at a distance: Foundations of distance education (3a. ed.). Boston, E.E.U.U: Pearson.

SIMONSON, M., SMALDINO, S., ALBRIGHT, M., \& ZVACEK, S. (2009). Teaching and learning at a distance: Foundations of distance education (4a. ed.). Boston, E.E.U.U.: Pearson.

SMALDINO, S. E., LOWTHER, D. L. \& RUSSELL, J. D. (2008). Instructional technology and media for learning (9a. ed.). Upper Saddle River, NJ, E.E.U.U. : Pearson Merrill Prentice Hall.

SORENSEN, C. K. \& BAYLEN, M. D. (2009). Adapting the seven principles of good practice to web-based instructional environment. En A. Orellana, T. L. Hudgins \& M. Simonson (Eds.), The perfect online course: Best practices for designing and teaching (pp. 69-86). Charlotte, NC, E.E.U.U.: Information Age Publishing.

SORIA, M. (2006). La capacitación en la administración de recursos humanos. Disponible en http:/www.rrhh-web.com/capacitacion.html

STROTHER, B. J. (2002). An assessment of the effectiveness of e-learning in corporate training programs. The International Review of Research in Open and Distance Learning, 1(3), 2-16.

TUNG, M., HUANG, J., KEH, H. \& WAI, S. (2009). Distance learning in advanced military education: analysis of joint operations course in the Taiwan military. Computer \& Education, 3(53), 653-666. 


\section{Apéndice A}

Pretest del curso básico de Microsoft Excel 2007 dirigida a un estudiante, para medir el nivel de aprendizaje alcanzado en un AVA.

Participante No.

1. Elabore la siguiente tabla en Microsoft Word 2007, correspondiente a las calificaciones de estudiantes del $6^{\circ}$ grado de educación primaria

\begin{tabular}{|l|c|c|c|c|}
\hline Nombre & Español & Matemáticas & Ciencias Naturales & Historia \\
\hline Participante 1 & 85 & 100 & 98 & 90 \\
\hline Participante 2 & 90 & 65 & 78 & 75 \\
\hline Participante 3 & 70 & 99 & 86 & 90 \\
\hline Participante 4 & 85 & 72 & 79 & 81 \\
\hline Participante 5 & 65 & 90 & 87 & 80 \\
\hline Participante 6 & 100 & 95 & 65 & 77 \\
\hline Participante 7 & 98 & 74 & 72 & 69 \\
\hline
\end{tabular}

2. Importe los datos de esta tabla a un libro de Microsoft Excel, y colóquelos en una hoja denominada Examen.

3. Coloque el siguiente encabezado (centrado) en la hoja de cálculo Examen. Además agregue una imagen prediseñada como un escudo de identidad.

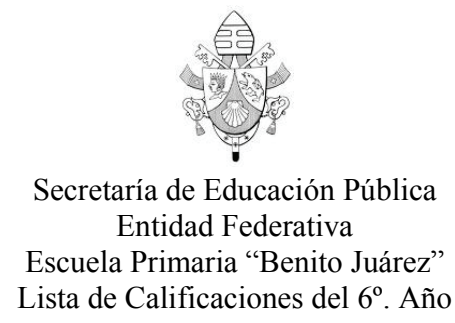

4. Agregue un registro más a la tabla. Este registro deberá referenciar su nombre y sus respectivas calificaciones.

5. Ordene los datos de la tabla por orden alfabético y coloque un sombreado al renglón que funge como encabezado.

6. Agregue una columna denominada promedio, y utilizando una fórmula de Microsoft Excel, obtenga el promedio de calificación por alumno. Adicionalmente utilice una función para insertar la fecha y hora del día. 


\begin{tabular}{|c|c|c|c|c|c|}
\hline Nombre & Español & Matemáticas & $\begin{array}{c}\text { Ciencias Natura- } \\
\text { les }\end{array}$ & Historia & Prom \\
\hline Participante 1 & 85 & 100 & 98 & 90 & 93 \\
\hline Participante 2 & 90 & 65 & 78 & 75 & 77 \\
\hline Participante 3 & 70 & 99 & 86 & 90 & 86 \\
\hline Participante 4 & 85 & 72 & 79 & 81 & 79 \\
\hline Participante 5 & 65 & 90 & 87 & 80 & 80 \\
\hline Participante 6 & 100 & 95 & 65 & 77 & 84 \\
\hline Participante 7 & 100 & 100 & 100 & 100 & 100 \\
\hline Participante 8 & 98 & 74 & 72 & 69 & 78 \\
\hline
\end{tabular}

7. Utilizando fórmulas matemáticas y estadísticas de Microsoft Excel, obtenga el promedio general de aprovechamiento del grupo, la media aritmética, la mediana, la moda y la desviación estándar.

8. Elabore una gráfica de columnas en 3D en Microsoft Excel. Coloque título de gráfico, título de eje X, y título de eje Y. Finalmente, genere una macro para este gráfico.

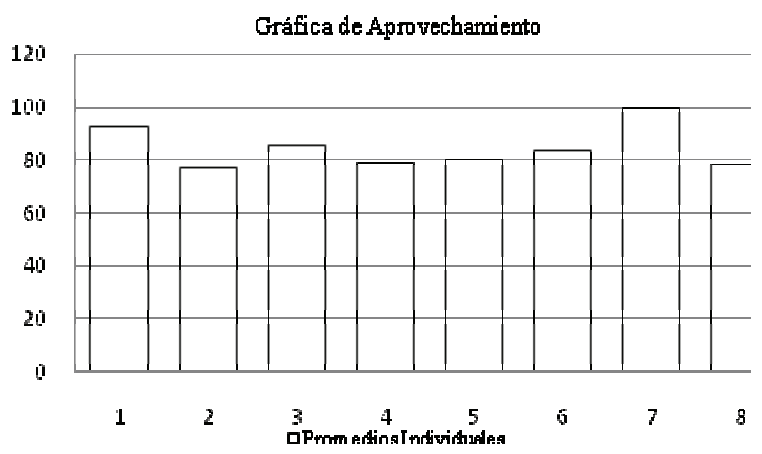

9. Utilizando un filtro, genere una nueva tabla que muestre sólo los registros que corresponden a los estudiantes que obtuvieron un promedio menor o igual a 80 .

\begin{tabular}{|c|c|c|c|c|c|}
\hline Nombre & Español & Matemáticas & $\begin{array}{c}\text { Ciencias } \\
\text { Naturales }\end{array}$ & Historia & Prom \\
\hline Participante 2 & 90 & 65 & 78 & 75 & 77 \\
\hline Participante 4 & 85 & 72 & 79 & 81 & 79 \\
\hline Participante 5 & 65 & 90 & 87 & 80 & 80 \\
\hline Participante 8 & 98 & 74 & 72 & 69 & 78 \\
\hline
\end{tabular}

10. Agregue un sello personal de edición y publique el libro creado en Excel, como una página web que permita ejecutarse a través de un explorador de la internet. 


\section{Apéndice B}

Postest del curso básico de Microsoft Excel 2007 dirigida a un estudiante, para medir el nivel de aprendizaje alcanzado en un AVA.

Participante No.

1. Elabore la siguiente tabla en Microsoft Word 2007, correspondiente a las calificaciones de estudiantes del $6^{\circ}$. grado de educación primaria.

\begin{tabular}{|c|c|c|c|c|}
\hline Nombre & Español & Matemáticas & $\begin{array}{c}\text { Ciencias Natura- } \\
\text { les }\end{array}$ & Historia \\
\hline Participante 1 & 85 & 100 & 98 & 90 \\
\hline Participante 2 & 90 & 65 & 78 & 75 \\
\hline Participante 3 & 70 & 99 & 86 & 90 \\
\hline Participante 4 & 85 & 72 & 79 & 81 \\
\hline Participante 5 & 65 & 90 & 87 & 80 \\
\hline Participante 6 & 100 & 95 & 65 & 77 \\
\hline Participante 7 & 98 & 74 & 72 & 69 \\
\hline
\end{tabular}

2. Importe los datos de esta tabla a un libro de Microsoft Excel, y colóquelos en una hoja denominada Examen.

3. Coloque el siguiente encabezado (centrado) en la hoja de cálculo Examen. Además agregue una imagen prediseñada como un escudo de identidad.

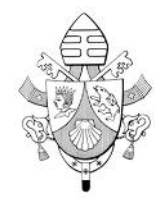

Secretaría de Educación Pública

Entidad Federativa

Escuela Primaria "Benito Juárez"

Lista de Calificaciones del $6^{\circ}$. Año

4. Agregue un registro más a la tabla. Este registro deberá referenciar su nombre y sus respectivas calificaciones.

5. Ordene los datos de la tabla por orden alfabético y coloque un sombreado al renglón que funge como encabezado.

6. Agregue una columna denominada promedio, y utilizando una fórmula de Microsoft Excel, obtenga el promedio de calificación por alumno. Adicionalmente utilice una función para insertar la fecha y hora del día. 


\begin{tabular}{|c|c|c|c|c|c|}
\hline Nombre & Español & Matemáticas & $\begin{array}{c}\text { Ciencias } \\
\text { Naturales }\end{array}$ & Historia & Prom \\
\hline Participante 1 & 85 & 100 & 98 & 90 & 93 \\
\hline Participante 2 & 100 & 95 & 65 & 77 & 84 \\
\hline Participante 3 & 85 & 72 & 79 & 81 & 79 \\
\hline Participante 4 & 70 & 99 & 86 & 90 & 86 \\
\hline Participante 5 & 90 & 65 & 78 & 75 & 77 \\
\hline Participante 6 & 100 & 100 & 100 & 100 & 100 \\
\hline Participante 7 & 65 & 90 & 87 & 80 & 80 \\
\hline Participante 8 & 98 & 74 & 72 & 69 & 78 \\
\hline
\end{tabular}

7. Utilizando fórmulas matemáticas y estadísticas de Microsoft Excel, obtenga el promedio general de aprovechamiento del grupo, la media aritmética, la mediana, la moda y la desviación estándar.

8. Elabore una gráfica de columnas en 3D en Microsoft Excel. Coloque título de gráfico, título de eje X, y título de eje Y. Finalmente, genere una macro para este gráfico.

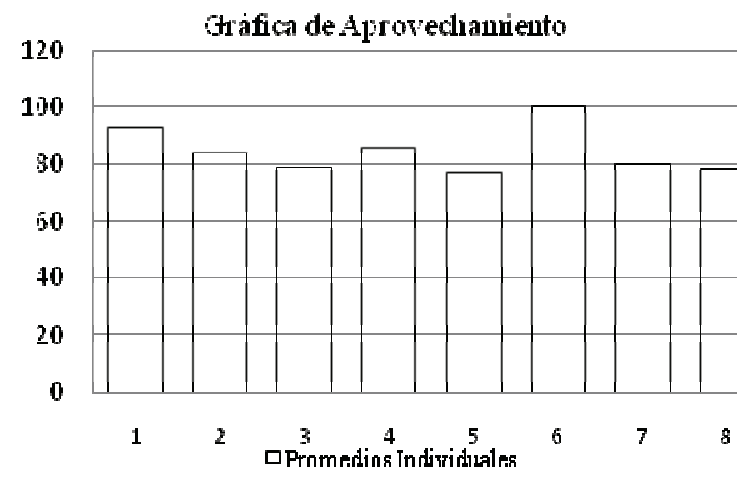

9. Utilizando un filtro, genere una nueva tabla que muestre sólo los registros que corresponden a los estudiantes que obtuvieron un promedio menor o igual a 80 .

\begin{tabular}{|c|c|c|c|c|c|}
\hline Nombre & Español & Matemáticas & $\begin{array}{c}\text { Ciencias Natu- } \\
\text { rales }\end{array}$ & Historia & Prom \\
\hline Participante 3 & 90 & 65 & 78 & 75 & 77 \\
\hline Participante 4 & 85 & 72 & 79 & 81 & 79 \\
\hline Participante 7 & 65 & 90 & 87 & 80 & 80 \\
\hline Participante 7 & 98 & 74 & 72 & 69 & 78 \\
\hline
\end{tabular}

10. Agregue un sello personal de edición y publique el libro creado en Excel, como una página web que permita ejecutarse a través de un explorador de la internet. 


\section{Apéndice C}

Cuestionario dirigido a un docente, para medir el nivel de satisfacción.

Participante No.

Instrucción: Conteste las siguientes preguntas acerca de la capacitación que recibió. Seleccione la opción que más refleje su opinión.

\begin{tabular}{|c|c|c|c|c|c|c|}
\hline & ¿Qué tan satisfecho está Ud., con & 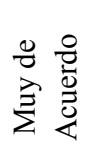 & 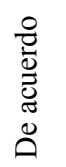 & $\begin{array}{l}\bar{\pi} \\
\stackrel{\Xi}{0} \\
Z\end{array}$ & 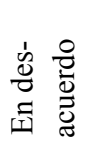 & 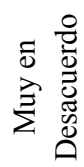 \\
\hline 1 & la organización del curso? & o & o & o & o & o \\
\hline 2 & el contenido del curso? & o & o & o & o & o \\
\hline 3 & la duración del curso? & o & o & o & o & o \\
\hline 4 & los materiales instruccionales? & o & o & o & o & o \\
\hline 5 & $\begin{array}{l}\text { las actividades académicas realizadas en el curso } \\
\text { (lecturas, prácticas, ejercicios)? }\end{array}$ & o & o & o & o & o \\
\hline 6 & los conocimientos y habilidades adquiridas? & o & o & o & o & o \\
\hline 7 & la disponibilidad y atención del instructor? & o & o & o & o & o \\
\hline 8 & $\begin{array}{l}\text { el proceso de retroalimentación llevado a cabo por } \\
\text { el instructor? }\end{array}$ & o & o & o & o & o \\
\hline 9 & $\begin{array}{l}\text { sus expectativas de aprendizaje en una modalidad a } \\
\text { distancia? }\end{array}$ & o & o & o & o & o \\
\hline 10 & $\begin{array}{l}\text { esta experiencia de capacitación en modalidad a } \\
\text { distancia? }\end{array}$ & o & o & o & o & o \\
\hline
\end{tabular}




\section{Apéndice D}

Cuestionario dirigido a un docente, para medir el nivel de aplicación del conocimiento, tiempo después de un curso de capacitación.

Participante No.

Instrucción: Conteste las siguientes preguntas acerca de la capacitación que recibió. Seleccione la opción que más refleje su opinión.

\begin{tabular}{|c|c|c|c|c|c|c|}
\hline & Con relación al curso de ME: & 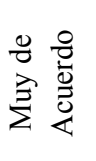 & 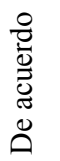 & $\begin{array}{l}\bar{\pi} \\
\stackrel{5}{0} \\
\text { 乙 }\end{array}$ & 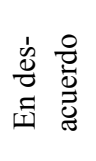 & 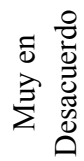 \\
\hline 1 & $\begin{array}{l}\text { ¿Los conocimientos adquiridos han tenido aplica- } \\
\text { ción en su ámbito laboral? }\end{array}$ & o & o & o & o & o \\
\hline 2 & $\begin{array}{l}\text { ¿Le ayudó a Ud., a mejorar el desempeño de sus } \\
\text { funciones? }\end{array}$ & o & o & o & o & o \\
\hline 3 & $\begin{array}{l}\text { ¿Le ayudó a Ud., a considerar nuevas formas de } \\
\text { trabajo? }\end{array}$ & o & o & o & o & o \\
\hline 4 & $\begin{array}{l}\text { ¿Generó en Ud., un incremento de motivación } \\
\text { hacia su trabajo? }\end{array}$ & o & o & o & o & o \\
\hline 5 & ¿Le sirvió a Ud., para su desarrollo personal? & o & o & o & o & o \\
\hline 6 & $\begin{array}{l}\text { ¿Le permitió a Ud., desarrollar algunas habilidades } \\
\text { adicionales? }\end{array}$ & o & o & o & o & o \\
\hline 7 & $\begin{array}{l}\text { ¿Le generó un cambio de actitud positiva hacia su } \\
\text { ambiente laboral? }\end{array}$ & o & o & o & o & o \\
\hline
\end{tabular}




\section{Apéndice E}

Cuestionario dirigido a directivos, para medir el nivel de resultados de sus colaboradores, después de un curso de capacitación.

Participante No.

Instrucción: Conteste las siguientes preguntas acerca de la capacitación que recibió. Seleccione la opción que más refleje su opinión.

\begin{tabular}{|c|c|c|c|c|c|c|}
\hline & $\begin{array}{l}\text { Con relación al curso sobre ME que su colaborador } \\
\text { realizó, considera Ud., que: }\end{array}$ & 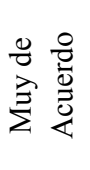 & 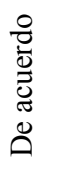 & 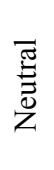 & 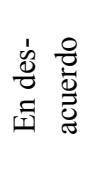 & 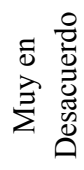 \\
\hline 1 & $\begin{array}{l}\text { ¿Los conocimientos adquiridos han generado } \\
\text { resultados positivos en su ámbito laboral? }\end{array}$ & o & o & o & o & o \\
\hline 2 & $\begin{array}{l}\text { ¿Le sirvió para mejorar el desempeño de sus fun- } \\
\text { ciones? }\end{array}$ & o & o & o & o & o \\
\hline 3 & ¿Le ayudó a considerar nuevas formas de trabajo? & o & o & o & o & o \\
\hline 4 & $\begin{array}{l}\text { ¿Le generó un incremento de motivación hacia su } \\
\text { trabajo? }\end{array}$ & o & o & o & o & o \\
\hline 5 & ¿Le ha servido para su desarrollo personal? & o & o & $\mathrm{o}$ & o & o \\
\hline 6 & $\begin{array}{l}\text { ¿Le permitió desarrollar algunas habilidades adi- } \\
\text { cionales? }\end{array}$ & o & o & o & o & o \\
\hline 7 & $\begin{array}{l}\text { ¿Le generó un cambio de actitud positiva hacia su } \\
\text { ambiente laboral? }\end{array}$ & o & o & o & o & o \\
\hline
\end{tabular}

\section{Correspondencia con los autores}

Marco Antonio CHAVEZ ARCEGA

Universidad Autónoma de Nayarit

Ciudad de la Cultura "Amado Nervo"

Tepic, Nayarit, México. C.P. 63155

Tel.: 52-(311)-2-18-00-60

E-mail:marcchav@nova.edu

\section{Liz ROMERO}

Nova Southeastern University

Fort Lauderdale, FL 33314 USA

750 NE 167th North Miami Beach, FL 33162

E-mail: lisbia@nova.edu 